\title{
Analysis on the Characteristics and Management Countermeasures of Truck Crashes on Zhejiang Expressways
}

\author{
Li Zheng Ming ${ }^{1 *}$, Jiang Tian Yue ${ }^{2}$, Zhu Jian Fang ${ }^{1}$, Wang Rui ${ }^{1}$ and Zhao Lili ${ }^{1}$ \\ ${ }^{1}$ Department of Safety Science and Engineering, Zhejiang University of Technology, China \\ ${ }^{2}$ School of Civil and Resources Engineering, University of Science and Technology, China
}

*Corresponding author: Li Zheng Ming, Department head of Safety Science and

Received Date: August 14, 2019

Engineering, Associate Professor, Zhejiang University of Technology, Hangzhou, China.

\section{Abstract}

Highway freight transport plays a very important role in China's transportation industry. Therefore, the characteristics and laws of highway cashes are analyzed, and targeted preventive measures and suggestions for improvement are put forward, which can be very good guidance for highway management in other regions.

Methods: A number of numerical analysis methods have been used to analyze 48861 truck cashes on Zhejiang expressways in the past five years. Various conversations with truck drivers suffice us to know the specific situations of the crash and suggestions for improvement of the management department.

Keywords: Urban public space;Truck crash; Characteristics; Management countermeasures

\section{Results}

The characteristics of the truck crashes are analyzed from the following three aspects-the road, the weather, and time, so as to survey the distribution under different conditions of the crash. Another two characteristics are revealed: there are many crashes caused by rear-end collisions on the highway, and expressway truck crashes lead to the serious consequence.

\section{Conclusion}

- $\quad$ The traffic crashes of trucks mostly occur in cities with better economic development. In the special road sections with complicated traffic conditions such as hubs, service areas, and tunnels, the ratio of expressway truck crashes is high;

- $\quad$ The distribution of truck crashes on expressways during the 24-hour period is significant. Fatal crashes often occur at night;

- From the safety management of expressway truck crash prevention, the improvement of the road environment can strengthen the monitoring process of road vehicles so that the truck drivers' safety awareness can be enhanced and eventually traffic crashes will be avoided.

\section{Introduction}

With the rapid development of our country's economy society, the construction of expressway is advancing by leaps and bounds, thus the leading freight transport vehicles bear the heavy transportation task. In 2015, the total freight volume of China's freight transport vehicles reached 31.5 billion tons and the cargo turnover reached 57955.72 billion tons [1]. Although the expressway only accounts for $2.4 \%$ of the total mileage of China's expressways, it has completed the turnover of $40 \%$ of the goods of the whole social business truck, which plays an important role in China's transportation industry [2-4]. Therefore, the characteristics and laws of expressway crashes are analyzed to propose crashes prevention and strategies from the point of view of safety management so as to put forward targeted preventive measures, which has a very good guiding significance for expressway management in other areas. 


\section{Methods}

We conducted a survey on the expressway management department and obtained the record of 48861 truck crashes on the expressway in Zhejiang Province in the past five years. In this paper, according to the cause of the crashes and the time and place, the number of casualties and other elements, the analysis of truck crashes rule from the three aspects-the road, the weather, and time [5], we analyze the characteristics of the crashes, and draw the distribution under different conditions of the crashes [6]. And we went to the expressway service area of Zhejiang Tonglu, Hangzhou, and had a face-to-face consultation with the truck drivers, to understand and verify the reasons for crashes and state, asked the driver the opinions of the expressway management, had formed a good crash prevention management measures [7].

\section{Results}

\section{Analysis on the distribution law of expressway truck crashes in zhejiang province}

Zhejiang Province is located on the southeastern coast of China and the southern wing of the Yangtze River Delta (in the east of Shanghai, China). It has 11 cities, covering an area of 101,800 square kilometers with a population of 55 million. There are about
5,900 kilometers of expressways. In 2015, the total economic volume of the whole province was 4288.6 billion yuan, and the road freight volume was 1225.47 million tons, accounting for $61 \%$ of China's total transportation volume, which was mainly based on the expressway truck transportation. During the 2011-2015, the number of deaths caused by traffic crashes on the expressway every year is about 324, the truck-caused deaths accounted for $69 \%$ of the total number of expressway crashes, which led to great economic losses and social impact.

\section{Distribution law of truck crashes location}

a. Distribution of truck crashes in urban areas: The distribution of average number of truck crashes in cities of Zhejiang Province is shown in Figure 1. The left main axis is the annual average number of crashes, and the second axis is the proportion. The number of crashes accounted for $12 \%$ and $6 \%$, which was divided into high-incidence cities, cities with a medium probability of crashes and low-incidence cities. Shaoxing, Hangzhou, Jinhua are the high-incidence cities, whereas Ningbo, Taizhou, Huzhou, Jiaxing, Quzhou, and Wenzhou are the cities with a medium probability of crashes, and Lishui together with Zhoushan are the low-incidence cities.

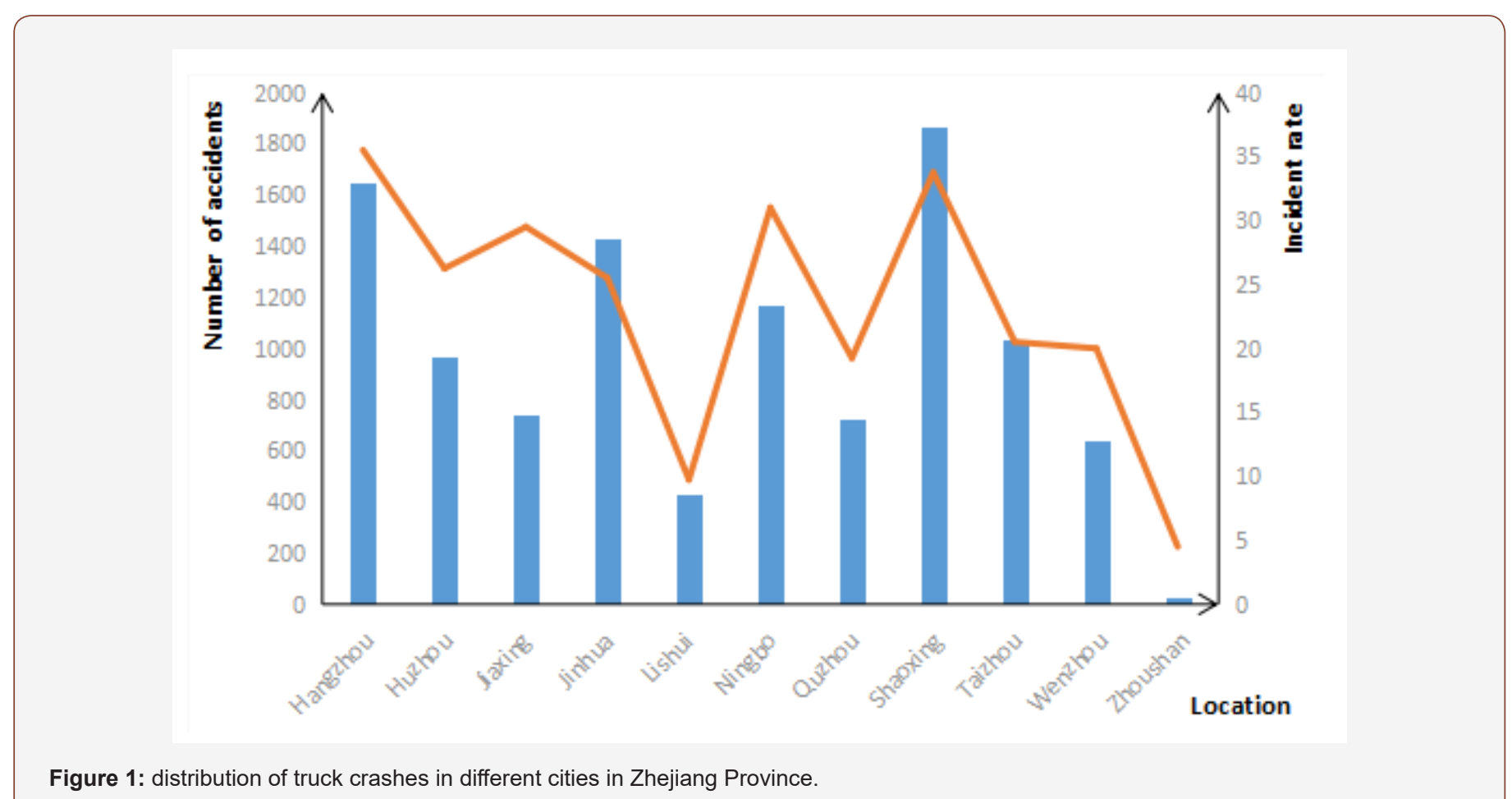

From the geographical location of the cities, the three cities of Hangzhou, Shaoxing and Jinhua are located in the inner core area of Zhejiang Province and have close connection with other urban areas. Hangzhou, as the economic core area of Zhejiang Province, its cargo transportation is also more closely related. Second, Ningbo, which is in the peripheral coastal city, is also a very economically developed area. However, the number of crashes is slightly lower due to the limited contact with the hinterland on both sides of the sea. The outlying cities of Huzhou, Jiaxing, Quzhou and Lishui are relatively underdeveloped economically, while the more economically developed Wenzhou is located in the southernmost part of Zhejiang Province with a coastal area of relatively low-speed traffic.

b. Distribution rules of high distribution of truck crashes: According to the statistics of the crashes in major expressways in Zhejiang Province from 2011 to 2015, the crashes are mainly in the various hubs, the entrance and exit of neighboring cities, the entrance and exit of service stations, the special locations of tunnels and so on. Among them, the study reveals that there are 
significant differences in the number of crashes in the direction of the expressway entering and leaving the city.

Taking the G56 Hangrui expressway as an example, Figure 2 shows the comparison chart of the number of crashes per kilometer in the direction of the expressway entering and leaving the city. Blue line is the number of crashes per kilometer in the direction of entering the city, near Hangzhou, the number of crashes higher and higher, up to $5.71 / \mathrm{km}$. Orange line is the number of crashes per kilometer in the direction of leaving the city, the number of crashes is under two per kilometer, and near the city. This pattern is especially pronounced at the end of a longer segment of the expressway, often referred to as the "tail-end effect". Along the same stretch, road design, facilities and traffic conditions of the two directions are basically the same, and the road traffic flows are also similar. Therefore, it is worth exploring the reasons for the difference in the number of truck crashes in the opposite direction.

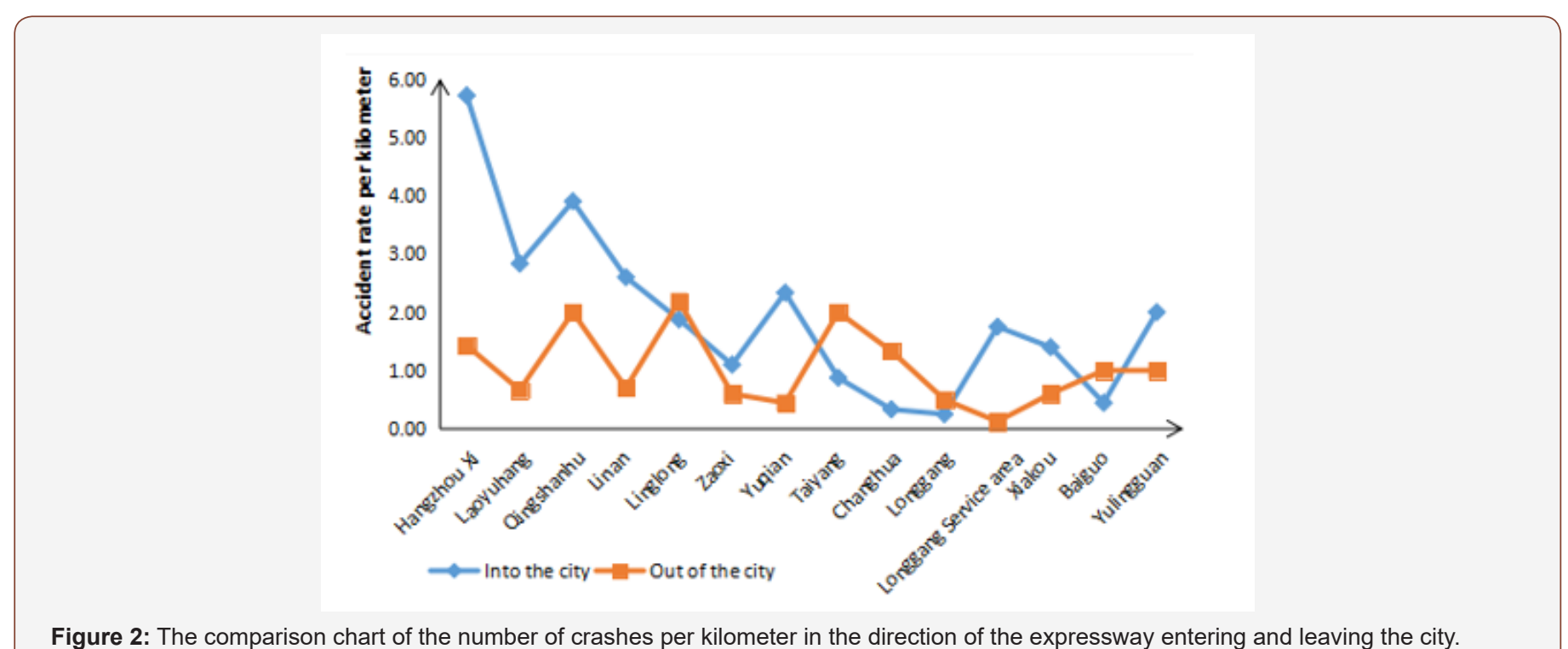

Figure 2: The comparison chart of the number of crashes per kilometer in the direction of the expressway entering and leaving the city.

Given that the vehicle conditions and the road conditions are the same, the main reason for the difference in the number of crashes is that the driver's driving behavior has been abnormal, and he has neglected his vigilance. Under normal circumstances, the driver will, after a long time of driving, enter the period of fatigue, and at the end of the expressway (which means close to the destination), both physically and mentally, becoming more relaxed at the same time. Moreover, the closer the driver is to the city, the heavier the traffic is, and the driver can't adapt to the change of the road traffic conditions, which led to the increase in the number of crashes in the direction of entering city, therefore, there is little difference in the number of crashes between the road in the direction of leaving city and the far road.

Time distribution laws of truck crashes: The influences of the time factor of expressway crashes are mainly reflected in the light traffic of driving, and the change of fatigue degree of the driver with the length of driving time [8-9].

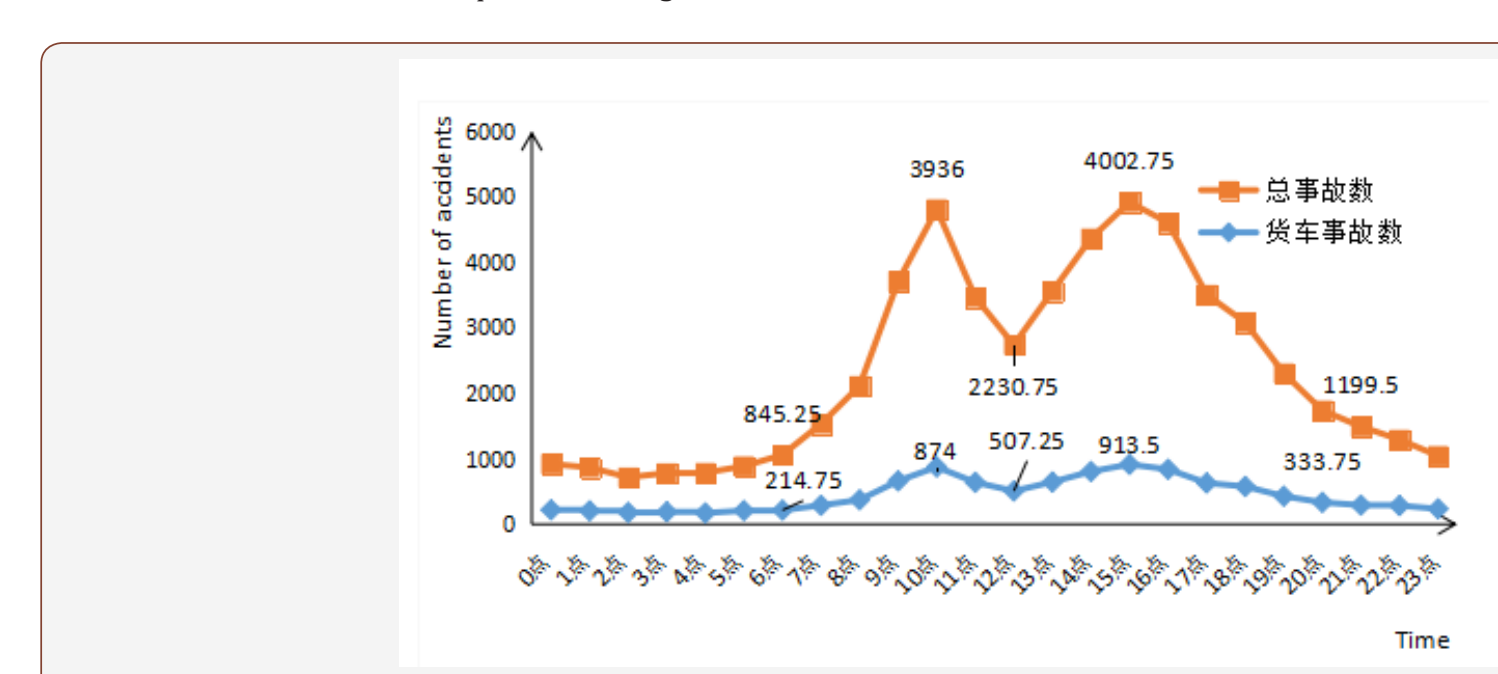

Figure 3: Comparison of 24-hour distribution of truck crashes and expressway crashes in Zhejiang Province.

A comparison chart of the 24-hour distribution of the number of truck crashes in Zhejiang Province and the number of expressway crashes in the past five years is shown in Figure 3. As can be seen from the trend of the curve on the map, the distribution of truck 
crashes and total crashes in the 24 hours is roughly the same, but the number of truck crashes in the total number of crashes has a greater difference between day and night.

During the daytime, the total crash rate of truck crashes is about $73 \%$, which is the high incidence of truck crashes, but it is relatively small and stable in the total number of crashes. Among them, the 10 o'clock in the morning and the 15 o'clock in the afternoon are the two peaks of the crash rate of trucks. During the two periods, the rate of crashes exceeds $8 \%$, and these two periods are also the peak periods of expressway traffic. At night driving segment, the crash rate of the truck crashes was all lower than that of the daytime driving segment, and between $1.61 \%$ and $3.12 \%$, the total incidence was $26.68 \%$. However, the average proportion of crashes in truck has risen from $22 \%$ in the daytime to $30 \%$, especially at 2am in the morning, which is more than $33 \%$. Figure 4 shows the trend of the average number of fatalities per truck per hour. As can be seen from the chart, the number of deaths per truck crash at night is 4 to 5 times that of daytime.

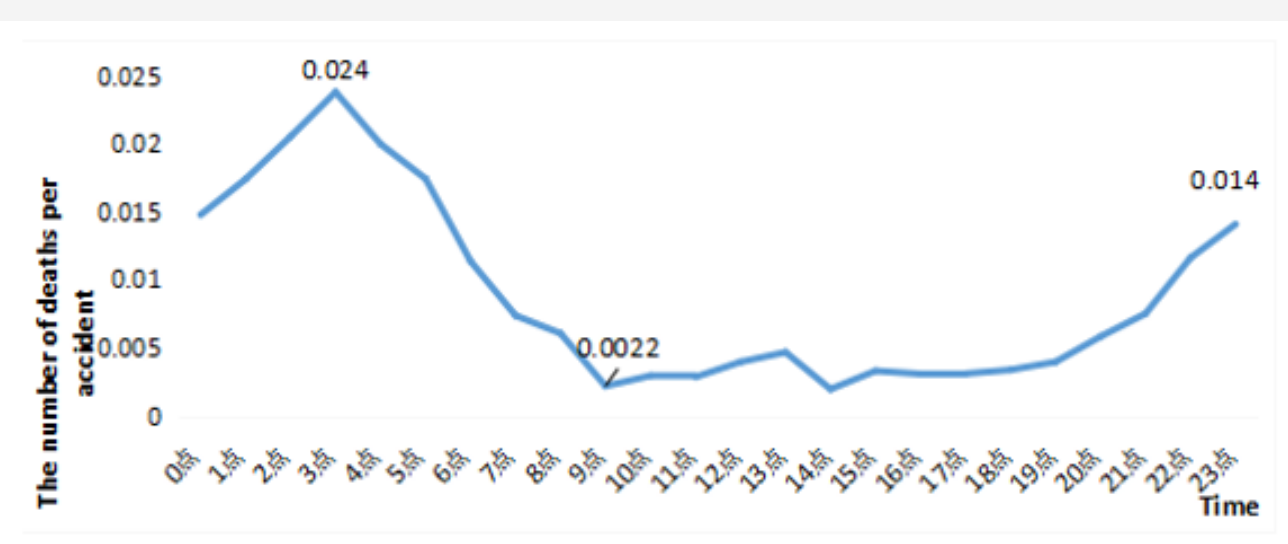

Figure 4: Distribution of average deaths per truck crash at various times.

In the daytime driving period, the high-speed traffic is generally high, and there are many types of passenger cars and trucks on the road, and the passenger and wagons have a high degree of mixture, the impact and interference between cars and vehicles are more serious, therefore, the crash rate is high, and the crash rate involving trucks is also relatively high. But because of driving during the day, the condition of the road going up is better than that at night, and the crash losses incurred are small and the severity is low. When driving at night, the road light is weaker, the driver's line of sight distance and field of vision is very poor, and the night driving vehicle, with the priority given to large trucks, mechanical performance of the vehicle has obvious difference with small vehicles. As a result, the casualties caused by the crashes are serious and the property losses are high.

In addition, the proportion of crashes caused by overloaded trucks at night increased significantly. The increase in overloaded trucks reflects the current bad habits of the trucking industry. Due to the weakening of the law enforcement supervision at night, the cargo companies can easily find loopholes and increase the carrying capacity of the trucks at night to improve the transportation efficiency.

Distribution characteristics of truck crashes and its relevant weather conditions: Changes in weather conditions will have a greater impact on road conditions, drivers' visibility, and vehicle performance, especially under extreme weather conditions, due to low visibility, road conditions, and vehicle technical conditions. Great changes can easily lead to misjudgments by the driver and frequent traffic crashes. Rain and snow weather reduced the visibility on the expressway, which badly affected the driver's view. The ground of expressways is easy to freeze, and with the low co-efficiency of friction, the snow disaster can easily affect the traffic safety facilities; The smoggy weather has a great influence on visibility and visual field, affecting the distance of sight. High temperature weather increases the failure rate of vehicles, which also affects the physiological and psychological state of the driver. In this way, the best driving conditions are sunny days or cloudy days, while rain and snow, especially snow days, have the worst driving conditions [10-11].

This article surveys the weather conditions of truck crashes in the past five years, as shown in Table 1. On expressways, snow days are the most prone to crashes. The incidence is close to $50 \%$, followed by sunny, cloudy and rainy days.

Table 1: Rate of all types of weather crashes.

\begin{tabular}{|c|c|c|c|}
\hline Weather & $\begin{array}{c}\text { Number of } \\
\text { Crashes }\end{array}$ & Days & $\begin{array}{c}\text { Incident } \\
\text { Rate }^{[12]}\end{array}$ \\
\hline Sunny & 7053.5 & 201 & $22.65 \%$ \\
\hline Snowy & 517.75 & 7 & $47.75 \%$ \\
\hline Cloudy & 1067.5 & 40 & $17.23 \%$ \\
\hline Rainy & 2241.5 & 117 & $12.37 \%$ \\
\hline
\end{tabular}

It can be seen that the casualty rate caused by truck crashes is quite different from the crash rate. From the casualty rate of truck crashes, the highest incidence of truck crashes occurred on snowy days, but the casualty rate was the lowest among several types of weather. Followed by cloudy and rainy days, the number of injuries per crash in these two types of weather was similar, followed by sunny weather. Foggy weather has the greatest impact on the field of vision, and it is the most vulnerable to injury. The analysis of the injuries and deaths of the people involved in the crashes is about 
the same as that of the injury rate. The difference is that the number of deaths per crash on sunny days is close to that of cloudy days, while on rainy days the number of deaths is lower, with the lowest number of deaths per crash on snowy days.

The weather conditions such as snow and fog have a relatively high incidence of bad weather crashes, but the number of crash casualties is mainly concentrated on sunny and cloudy weather conditions with good driving conditions. According to the statistics, more than $70 \%$ of crashes on snowy days are caused by improper operations, and crashes caused by illegal driving are less than $15 \%$. It is explained that the driving conditions of snow day and icy roads have higher operating requirements and higher levels for drivers to drive at high speed, and drivers will be more careful when driving, so as to avoid dangerous driving behaviors, and attention is also more concentrated. Therefore, most of the crashes that occurred were caused by insufficient driver levels. On the contrary, on rainy days, driving conditions are better than those of snow days, and the proportion of illegal driving is much higher, especially for speeding and overloading, which is twice as high as the snow day. This has a lot to do with the driver's psychological situation. Drivers with longer driving experience will be overconfident, ignoring the dangerous situation of obstructed vision and low ground friction coefficient caused by rain. This leads to higher severity of crashes and will cause more serious casualties.

\section{Analysis of characteristics of other truck crashes:}

a. More rear-end collisions on the expressways: The truck crashes in the expressways in Zhejiang Province mainly consisted of rear-end collisions, which accounted for $58 \%$ of all crashes. In these rear-end collision crashes, there are also many consecutives rear-end crashes on the expressways. For example, a total of 39 rear-end collisions occurred on January 1, 2014, and 17 crashes were consecutive rear-end collisions. In particular, two of them were caused by failure to evacuate after the crashes or set up related signs (Figure 5).

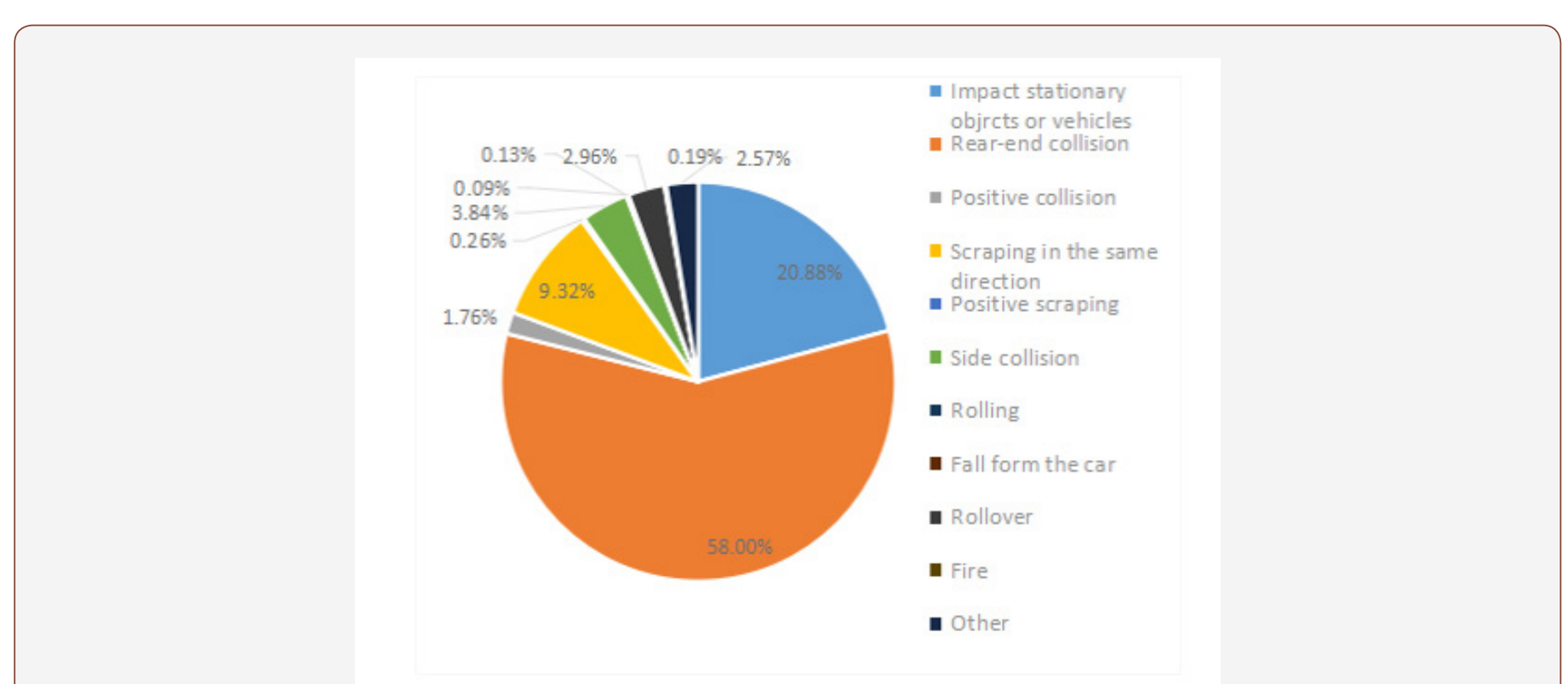

Figure 5: Zhejiang Expressway Truck crashes Form Distribution.

b. Serious consequence of expressway truck crashes: In Zhejiang Province, the number of expressway truck crashes in 2011-2015 accounted for $23.52 \%$ of the total number of crashes, but the economic losses and casualties caused by it were particularly serious [12]. Only the proportion of vehicle damage and the number of crashes is close, direct economic losses and deaths accounted for about $75 \%$ of all crashes, while the injured are more than 50\%. This indicates that crashes involving trucks often cause a large number of casualties and economic losses, and the crashes severity is particularly high.

\section{Expressway Freight Car Safety Management Countermeasures}

\section{Improve expressway traffic environment:}

- It's a necessity to completely improve the road surface environment of the expressway, optimize the road alignment, and reduce the slope of the road. In some of the uphill sections, climbing lanes can be set up specifically for low-speed trucks, increase the road friction and enhance the strength of the fence; a prescribed number of fire-fighting facilities should be installed in the tunnel.

- Road maintenance units are required to carry out road maintenance operations in a timely manner, and road administration and traffic police shall be coordinated to ensure the normal and orderly opening of maintenance road sections and maintain the order of expressway maintenance operations to prevent crashes.

- It's also a great necessity to scientifically allocate warning signs on the road surface, add simulated policemen, and mark information on crashes-prone road sections to promptly remind drivers of driving safety. 


\section{Strengthen the supervision of the entire process of truck driving:}

- Using information technology to install and implement remote location supervision in the trucks, all long-distance transportation vehicles are required to install GPRS positioning system, and the safety management personnel of the cargo transportation company shall supervise whether the route of the vehicle is correct or not, by setting a violation reminder function. For vehicles that are continuously running for more than two hours, they must remind the driver to rest in the service area and control fatigue while driving and correct drivers' unsafe behavior in time.

- In addition to installing GPRS positioning and monitoring systems for dangerous chemicals transport vehicles, remote video surveillance systems should also be installed in the cab, the driver's driving behavior was photographed and recorded. When unsafe driving behavior was found, the driver was recorded and punished.

- It's of great urgency to give full play to the supervision and management functions of the road network security information system, and timely monitor unsafe acts such as fatigue driving.

- Improve the automobile safety laws and regulations system, establish internationally accepted automobile technical regulations, enforce the law strictly, and use legal means to restrict drivers' unsafe behavior.

\section{Improve driver safety awareness education:}

- $\quad$ Carry out multi-channel expressway safety management publicity education, safety knowledge learning and crashes warning education activities, improve the self-restraint ability of truck drivers, strictly control the speed of vehicles, and conduct civilized driving. Improve the driver's ability to handle emergency risks.

- $\quad$ Revise and improve traffic safety regulations, advocate heavy penalties, impose severe penalties for violations of law, increase the seriousness of law enforcement, implement government safety incentives for drivers who have no violations throughout the year, and reduce vehicle insurance rates appropriately, and form a kind of insurance privilege, reward and punishment clear mechanism.

- The driver is required to strengthen the vehicle performance inspection. For the over-used trucks, the vehicles should receive more rigorous and careful inspections before entering the expressway to avoid hidden problems.

\section{Conclusion}

- Overall, the traffic crashes of trucks in Zhejiang Province mostly occurred in cities with better economic development.
However, the severity of crashes was negatively related to the economic level; in the special road sections with complicated traffic conditions such as hubs, service areas, and tunnels, the incidence of expressway truck crashes is high.

- The distribution of truck crashes on expressways during the 24-hour period is significant. The occurrence of crashes has two peaks, 10:00 and 15:00. Fatal crashes often occur at night.

- $\quad$ From the safety management of expressway truck crashes prevention, the casualty rate is due to driver's fatigue driving, illegal driving, improper operation, and illegal parking is high. Take the improvement of the road environment, strengthen the process monitoring of road vehicles, improve driver safety awareness, and finally prevent truck crashes.

\section{Acknowledgement}

This research was supported by NSFC 51674224 .

\section{Conflict of Interest}

No conflict of interest.

\section{References}

1. (2016) Ministry of Transport. 2015 Communique for the development of traffic and transportation industry [OL].

2. (2014) Meng li. Big data uncovering the truth of freight cars in China's Freeway [OL].

3. (2016) Zhejiang Province Bureau of Statistics, 2015 Zhejiang province keeps growing of freight volume in the whole society.

4. (2015) Liu Yongta, 4275 people died in Zhejiang's 2015 traffic crashes.

5. Golob TF, Recker WW (2003) Relationships Among Urban Freeway Accidents, Traffic Flow, Weather and Lighting Conditions. Journal of Transportation Engineering 129(4):342-353.

6. Wang Yuhan (2011) Road traffic accident analysis method [D]. Jilin University.

7. Chen GX, Sieber WK, Lincoln JE, Birdsey J, Hitchcock EM, et al. (2015) NIOSH national survey of long-haul truck drivers: Injury and safety[J]. Accident; analysis and prevention 85: 66-72.

8. Chen GX, Collins JW, Sieber WK, Stephanie G Pratt, Rosa L Rodríguez Acosta, et al. (2015) Vital signs: seat belt use among long-haul truck drivers--United States, 2010. [J]. Mmwr Morbidity \& Mortality Weekly Report 64(8): 217-221.

9. Golob TF, Regan AC (2003) Truchk-inbolved crasheses and traffic levels on urban freeways[J]. University of California Transportation Center Working Papers.

10. Dong Keyang, Lu Baichuan (2008) In fluences of Bad Weather to Movements of Vehicles [J] Journal of Chongqing Jiaotong University (Social Science Edition) 6: 24-26.

11. Christopher K Strong, Zhirui Ye, Xianming Shi (2010) Safety Effects of Winter Weather: The State of Knowledge and Remaining Challenges[J]. Transport Reviews 30(6): 677-699.

12. Vijaya RB, Sreedhar M (2013) Evaluation of Reliability and Traffic Accident Frequency Rate by Using System Reliability Model-A Simulation Approach[J]. International Journal of Statistics \& Analysis 3(1). 